\title{
Electromagnetic fields (EMF) exposure
}

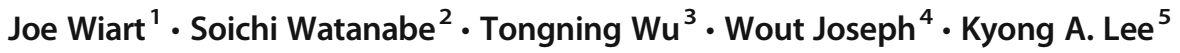

Published online: 14 January 2019

(C) Institut Mines-Télécom and Springer Nature Switzerland AG 2019

The electromagnetic fields-whatever their frequency bands - are increasingly being used in wireless communications that nowadays are used in almost every aspect of our daily life. Over the past 30 years, wireless communication systems have been increasingly used; the versatile use of new mobile phones and the emergence of all-pervasive wireless communication systems, such as machine-to-machine communication, are strengthening this tendency.

Despite this tremendous use and as shown in several surveys like the Euro-barometer published by the European Commission (ref 2010), the general public still has a particular attention to the electromagnetic fields (EMF) exposure induced not only by wireless networks and devices but those induced by sensors and smart meters. To date, no adverse health effects of the EMF, linked to these applications, have been established. Large efforts worldwide have been carried out since starting the WHO's International EMF Project in 1996. To protect the public against known health effects of electromagnetic fields, limits have been established. ICNIRP (International Commission on Non-Ionizing Radiation Protection) has defined basic restrictions that limit the specific absorption rate (SAR), expressed in Watt/kg, and characterize the RF human absorption. ICNIRP also defined reference levels that are limiting the incident field strength to the level inducing an exposure compliant with the basic restrictions.

$5 \mathrm{G}$ networks are being commercially deployed; they are a step toward increasing throughputs and huge amounts of data transferred every second and everywhere on the planet. They

Joe Wiart

joe.wiart@telecom-paristech.fr

Télécom ParisTech, Paris, France

2 NICT, Tokyo, Japan

3 CAICT, Beijing, China

4 Ghent University/IMEC INTEC-WAVES, Ghent, Belgium

5 ETRI, Daejeon, South Korea also bring new challenges for the EMF exposure management. This special issue addresses some of these challenging problems. The issue's papers are covering a wide range of issues from simulations to measurements as well as from deterministic to statistical approaches. In addition, an invited paper is dealing with sociology and risk perception.

After a thorough reviewing process where every paper has been evaluated by at least two experts, 11 papers have been accepted for this special issue including two invited papers. Reviewers' comments were very helpful first to select the most significant contributions as well as to improve the content, quality, and presentation of the accepted papers. Hereafter, a summary of each paper in this special issue is provided.

The first two papers are invited papers. The first one from Laura Draetta is dealing with "The social construction of a health controversy, the case of electricity smart meters in France." It presents a sociological study of the public controversy over electricity smart meters in France. It analyzes how this controversy has emerged and grown. It highlights the public, multidimensional, and healthfocused nature of the controversy and shows how this controversy extends beyond discussion of the intrinsic qualities of the smart meter to fuel reflection on the social dimension of the rollout.

The second one from Akimasa Hirata and colleagues is entitled "Setting Exposure Guidelines and Product Safety Standards for Radio-Frequency Exposure at Frequencies Above $6 \mathrm{GHz}$." This paper deals with setting EMF exposure international protection guidelines - which are currently under revision - and is focusing on frequencies above 3 or $10 \mathrm{GHz}$ where the $5 \mathrm{G}$ wireless communication system will be deployed soon. In this review, the authors focused on three issues to be considered in the next revision of the exposure guidelines: (i) the averaging area of the incident power density, (ii) the transition frequency at which the metric is changed from the specific absorption rate to the incident power density, and (iii) the exposure averaging time. 
The three next papers are investigating the real radiofrequency exposure linked to present and future networks:

The first one from Congsheng Li and colleagues is dealing with "Numerical Evaluation of Human Exposure to 3.5 GHz Electromagnetic Field by considering the 3GPPlike Channel Features." The authors evaluated the EMF exposure of the 3.4-3.6 GHz frequency band by taking into account the channel features. Two exposure scenarios were reconstructed according to the technical specification on channel modeling from the 3rd Generation Partnership Project. Toward such an objective, the authors used the equivalent source principle and the finite-difference-timedomain method to calculate the RF energy specific absorption (SA) using three human models.

The second one from Taghrid Mazloum and colleagues is entitled "RF-EMF exposure induced by mobile phones operating in LTE small cells in two different urban cities." This paper studies the RF exposure induced by mobile phones operating in LTE small cells in two different urban cities. These low-powered small cells are used for network densification; they improve network performances but they also influence the human RF exposure. To characterize the influence of network architecture on RF-EMF exposure induced by LTE and in particular the contribution of the uplink and downlink, the authors performed measurements in two different urban cities in France and the Netherlands.

The third one from Imtiaz Nasim and Seungmo Kim is entitled "Mitigation of Human EMF Exposure in Downlink of 5G." In this paper, the authors analyze the exposure induced by cellular communications operating at high frequencies above $6 \mathrm{GHz}$. Performing simulations, the authors investigated the influence of the downlink on human exposure and suggested that the downlink of a Fifth Generation Cellular Mobile Communications (5G) system can generate significantly higher power density (PD) and specific absorption rate (SAR) than the previous generations of wireless systems.

The next paper is dealing with the RF associated to a quite new technology called the wireless power transfer. Authored by Satoshi Shimoyama and colleagues, the paper is entitled "New Extrapolation Expression for Exposure Evaluation inside a Human-Equivalent Liquid Phantom in the Vicinity of Wireless Power Transfer Systems." This paper studies safety against electromagnetic exposures due to the wireless power transfer (WPT) systems operating at MHz-band frequencies. Compliance tests have to be conducted; however, accurate measurements of SAR are difficult and extrapolation methods are used to determine the internal electric field and relative SAR. In this paper, the authors proposed a new extrapolation expression based on the characteristics of electromagnetic fields inside the phantom. The validity of the proposed expression is analyzed both numerically and experimentally.

The three next papers are investigating the exposure assessment using measurement:
The first paper authored by Reza Aminzadeh and colleagues is entitled "The Effect of Antenna Polarization and Body Morphology on the Measurement Uncertainty of a Wearable Multi-Band Distributed Exposure Meter." This paper studies the effect of antenna polarization on measurement uncertainty of a multi-band body-worn distributed exposure meter. This system consists of eight nodes and is calibrated on four human subjects for simultaneous measurement of the incident power density in four frequency bands. The authors investigated the influence of the body on the results.

The second paper authored by Milica Popovic and colleagues is entitled "Experimental Analysis of Individual EMF Exposure for GSM/UMTS/WLAN User Devices." This paper investigates the exposure to electromagnetic fields (EMF) originating from a user device when using different services over WLAN, GSM, and UMTS technologies in different radio conditions. The exposure analysis performed using triggered network reports, external measurements performed in a live network, and simulation results showed a strong dependence on individual EMF exposure on wireless technology, radio conditions, and service used.

The last paper of this group of three is authored by Takahiro Iyama and colleagues and is dealing with "Whole-Body Average SAR Measurement Using Flat Phantoms for Radio Base Station Antennas and Its Applicability to Adult and Child Human Models." This paper discusses the effectiveness of SAR measurement procedure for radio base station antennas using flat phantoms considering Japanese human models based on numerical simulations with validation of wholebody average SAR (WBSAR) measurement. Using computational results, the authors showed the SAR measurement procedure in the IEC leads to overestimated WBSAR compared to those in Japanese anatomical human models. In addition, to reduce the measurement time and simplify the post-processing, this paper introduces an SAR-measurement procedure based on the two-dimensional SAR distribution around the surface of the bottom of the phantom and the onedimensional exponential decay of the SAR distribution in the direction of the phantom depth.

The last two papers are investigating the use of a statistical method in dosimetry often known as stochastic dosimetry:

The first paper of this group is "Statistical model of the human RF exposure in Small cells environment" and is authored by Amirreza Chobineh and colleagues. This paper investigates the use of statistical model to assess the global exposure (from user equipment and base stations) linked to small cell architecture. In such an environment, the propagation channel becomes highly variable and strongly susceptible to environmental factors. The authors propose an innovative statistical path loss model based on measurements; they use this model to assess global exposure of the adult proportion of a population. 
The second and last paper of the group and of this special issue is investigating the use of advanced surrogate modeling in numerical dosimetry. The paper is entitled "Children exposure to femtocell in indoor environments estimated by sparse low rank tensor approximations" and is authored by Emma Chiaramello and colleagues. This paper investigates the exposure assessment using surrogate models based on sparse low-rank tensor approximation method (sparse LRA). The authors are using this approach to assess the exposure of an 8-year-old child to a femtocell operating at $2600 \mathrm{MHz}$, both (child and source) freely located in random positions in an indoor environment. The surrogate models were used for fast estimating the specific absorption rate (SAR) in all the possible positions of femtocell and child.
Acknowledgments The guest editors would like to express their deep appreciation to the editor-in-chief, Prof. Guy Pujolle, as well as to the associate editor-in-chief, Nicolas Puech, for giving them the opportunity to publish this special issue. The guest editors also thank the managing editor, Alexia Kappelmann, as well as the journal editorial staff for their continuous support during the process of this publication. Lastly, the guest editors would like to express their thanks to all the authors for submitting quality articles and the reviewers for helping in the selection of papers and improving the accepted papers.

\section{Publisher's Note}

Springer Nature remains neutral with regard to jurisdictional claims in published maps and institutional affiliations. 